\title{
une classification géotechnique des craies du nord utilisée pour l'étude de stabilité des carrières souterraines
}

\author{
par
}

J. Bonvallet

Docteur - Ingénieur*

\begin{abstract}
L'étude de la stabilité d'un ouvrage souterrain existant se traduit en grande partie par l'appréciation du coefficient de sécurité statique défini communément comme le quotient de la charge admissible par le matériau, par les sollicitations régnant dans le massif.

Cependant, la détermination de la grandeur admissible $\mathrm{R}$ pose deux types de problèmes:

- I'hétérogénéité des milieux naturels se traduit par la dispersion des grandeurs mécaniques qui obéissent à une loi de distribution affectée d'une valeur moyenne et d'un écart type.

- la notion de grandeur admissible est mal définie mais est aussi relative et varie avec le genre de sollicitations (statique, dynamique) et leur durée (momentanées ou définitives).

Nous naborderons ici que le premier problème, dans le cadre des exploitations par chambres et petits piliers abandonnés du Nord de la France, situées dans les craies sénoniennes et turoniennes [2].
\end{abstract}

\section{Introduction}

Au cours de ces dernières années, peu de matériaux ont suscité autant d'études que la craie. L'importance quantitative de ce faciès dans le Bassin Parisien, dont il est l'un des principaux constituants, explique en partie les préoccupations convergentes de nombreux géotechniciens opérant dans le génie civil ou dans les travaux souterrains $[4,5]$.

Cependant, l'ambiguité même du terme craie, qui désigne des faciès déposés pendant près de 25 millions d'années, explique les déboires parfois rencontrés par les praticiens et la grande variabilité des grandeurs physiques et mécaniques qui lui ont été attribué.

Outre cette diversification des faciès, il semble bien que les outils habituellement utilisés en identification géomécanique n'aient qu'une efficacité relative pour caractériser cette roche que l'on a pu qualifier d'évolutive.

L'essai de résistance à la compression simple, essentiel dans tout calcul de stabilité d'ouvrage souterrain, apparait particulièrement dispersé et très sensible aux conditions expérimentales, notamment au degré de saturation [1, 3]. D'une façon générale, l'expérimentateur est conduit à multiplier des essais onéreux afin d'obtenir une valeur moyenne qui de surcroit ne constitue pas nécessairement un bon estimateur (fig. 1).

L'étude des corrélations entre paramètres physiques et mécaniques peut nous en affranchir. Pour ce faire, deux grandes voies sont envisageables:

La première expérimentée avec succès sur les craies blanches par Tallon [6] consiste à corréler texture et caractéristiques mécaniques. Cette méthodologie peut se décomposer en 4 parties.

- Observation des échantillons au microscope électronique à balayage (MEB). Cet examen exclusivement morphologique met en évidence la nature des liaisons intergranulaires qui conditionnent la résistance mécanique d'un milieu poreux interconnecté.

Fig. 1 Lezennes. Craie saturée $\emptyset$ 76. Elancement 2. Histògramme de la résistance à la compression

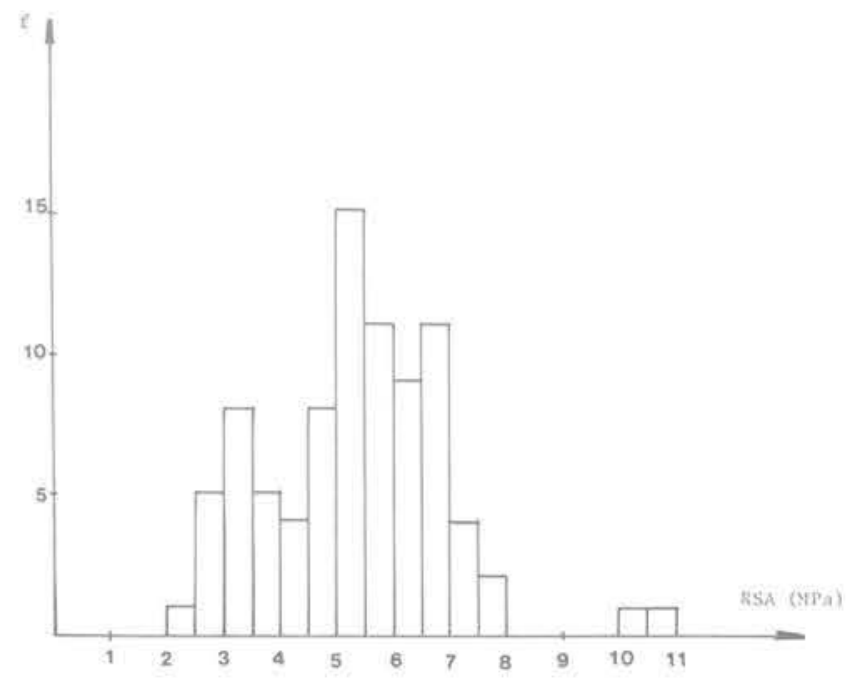


- Quantification de la texture par un nombre d'essais indirects minimum : vitesse des ondes longitudinales à l'état sec, porosité mesurée par porométrie au mercure et poids spécifique sec.

- Mise au point d'un essai de résistance mécanique en liaison avec le comportement du matériau en chantier de Génie Civil. Cet essai de référence est l'essai de vibrobroyage de Struillou, légèrement modifié.

- Etude de corrélations multiples entre paramètres de texture et paramètres mécaniques.

A l'issue de cette étude l'auteur prévoit le comportement en terrassement de la craie.

Cette démarche est très séduisante, par ses aspects successivement naturaliste, puis mécaniste et enfin mathématique: cependant, sa validité est conditionnée par la constitution quasi monominérale de la craie blanche oủ la texture est l'aspect déterminant. Son application éventuelle à nos problemes suscite deux remarques:

- les faciès rencontrès dans le cadre de nos études varient dans un vaste domaine où les craies dites "atypiques" sont largement représentées (craies glauconieuses, phosphatées, siliceuses, etc...). Dans ce cas, la texture n'est pas nécessairement le paramètre essentiel, ou du moins n'explique pas tout.

- les essais mis en jeu pour quantifier la texture et notamment l'essai de porométrie au mercure, mais aussi l'observation morphologique au MEB, sont du ressort des spécialistes et, par là-même, fort coûteux.

Aussi, proposons-nous une deuxième vole d'accès à la connaissance géotechnique des craies, basée sur une étude statistique multidimensionnelle se référant "a posteriori" aux données naturalistes pour l'interprétation.

\section{But et définition de l'étude}

Cette étude utilise les techniques d'analyse des données usuelles :

- méthode de classification automatique

(nuées dynamiques).

- analyse en composantes principales.

- analyse factorielle discriminante.

L'analyse des données présente l'avantage de ne faire aucune hypothèse "a priori" et cherche à décrire, classer et éventuellement réduire les données.

Nous recherchons :

- une classification basée sur des paramètres simples.

- des partitions finales les plus distinctes et les plus stables possibles, quel que soit l'outil utilisé.

La démarche adoptée comporte 4 volets, schématisés comme suit

\begin{tabular}{|c|c|}
\hline Etapes & Outil \\
\hline $\begin{array}{l}\text { 1- Qualification et quantification } \\
\text { des populations prélevées par } \\
\text { des essais simples, rapides, peu } \\
\text { onéreux. }\end{array}$ & $\begin{array}{c}\text { Essais courants } \\
\text { d'identification } \\
\text { physique }\end{array}$ \\
\hline $\begin{array}{l}2 \text { - Quantification de la résistance } \\
\text { mécanique des populations. }\end{array}$ & $\begin{array}{l}\text { Résistance à la } \\
\text { compression simple }\end{array}$ \\
\hline $\begin{array}{l}3 \text { - Classification, sans "a priori", } \\
\text { débouchant sur des partitions } \\
\text { stables, utilisant les paramétres } \\
\text { physiques. }\end{array}$ & $\begin{array}{l}\text { Analyse } \\
\text { des données }\end{array}$ \\
\hline $\begin{array}{l}\text { 4- Etude des corrélations entre } \\
\text { variables physique et variables } \\
\text { mécanique, au sein de chaque } \\
\text { partition. }\end{array}$ & $\begin{array}{l}\text { Analyse des } \\
\text { données } \\
\text { statistiques } \\
\text { mathématique }\end{array}$ \\
\hline
\end{tabular}

\section{Qualification et quantification des paramètres physiques}

Le critère de sélection d'un paramètre est la simplicité de l'essal et son faible coût. II doit pouvoir être mis en œuvre dans n'importe quel laboratoire.

L'analyse porte sur 22 blocs provenant de 20 sites. En moyenne il a été façonné 20 éprouvettes dans chaque bloc. ces éprouvettes étant par la suite dissociées en 2 lots. La méthodologie adoptée est représentée sur le tableau suivant:

\begin{tabular}{l|c|c|c}
\cline { 2 - 4 } & lot H & lot S & \\
\hline $\begin{array}{l}\text { Mise à saturation } \\
\text { Pesage }\end{array}$ & oui & oui & \\
$\begin{array}{l}\text { Mesure de VSA (1) } \\
\text { oui }\end{array}$ & oui & oui & Mesure de VSE \\
$\begin{array}{l}\text { Sechage } \\
\text { Mesure de VSE (1) }\end{array}$ & non & oui & oui \\
Pesage & non & oui & PVS \\
\hline Essai de compression & oui & oui & $\begin{array}{c}\text { Détermination de } \\
\text { RSA-RSE-ESA-ESE (1) }\end{array}$ \\
\hline
\end{tabular}

(1) Dans tout l'exposé PVS, W, VSE, VSA désignent respectivement : le poids volumique sec, la teneur en eau à saturation, la vitesse des ondes longitudinales à l'état $\mathrm{sec}$ et à l'état saturé, RSE, RSA, ESE, ESA désignent respectivement la résistance à la compression instantanée, à l'état sec ou saturé et les modules de déformation correspondants.

\section{Qualification : variable pétrographique}

Les craies du Nord appartiennent au Turonien et à la base du Sénonien (Coniacien). Nous avons distingué les craies blanches sénoniennes des craies blanches turoniennes riches en silex cornus, "a posteriori", ayant remarqué des différences notables tant du point de vue physique que du point de vue mécanique. En ce qui concerne les craies glauconieuses, 2 pôles ont été individualisés suivant que la glauconie était diffuse ou litẻe en feuillets visibles à l'œil nu. Cette discrimination globale concerne les bancs entiers, correspondants à des niveaux exploités. Dans le cadre d'une étude de stabilité, il n'est pas utile d'aller plus loin dans la distinction des faciès. Les niveaux phosphatés et indurés, proches de la limite turono-coniacienne ou les niveaux argileux du Toronien n'ont pas une influence notable du point de vue mécanique et ne seront pas étudiés.

Remarque 1 : La teneur en eau á saturation W est un indice de porosité. Pour un milieu poreux à structure punctique comme la craie, il est clair que la porosité vraie ne peut être déterminée qu'à l'aide de méthodes sophistiquées.

Remarque 2: L'impulsion génère aussi des ondes de cisaillement ou ondes transversales dont on ne tient pas compte pour trois raisons :

1 - Les ondes transversales ont une célérité VT toujours inférieure à celle VL des ondes longitudinales. Elles ne parasitent donc pas la mesure.

2 - Leur prise en compte nécessite un oscilloscope et un expérimentateur averti, ce qui est contraire aux impératifs fixés.

3 - En ce qui concerne la craie, la corrélation VL - VT est toujours très bonne VL $=1,6 \mathrm{VT}$ ( $\rho=0,937)$. (Tallon, 1976).

\section{Quantification des caractéristiques mécaniques}

Dans l'état actuel de nos connaissances, le paramètre pris en compte lors de l'étude de stabilité découle de la résistance à la compression simple.

Les conditions expérimentales sont les suivantes :

- éprouvettes cylindriques aux dimensions normalisées, sèches ou totalement saturées.

- presse de 20 tonnes a vitesse de chargement de 50 $\mu \mathrm{m} / \mathrm{mn}$. 
Suivant le degré de saturation on mesure donc, la résistance a la compression instantanée, à l'état sec ou saturé, et le module déformation correspondant.

Remarque : Des résultats complémentaires, issus de 341 essais de résistance à la compression simple, effectués à partir de 8 sondages dans 5 sites, ont été confrontés aux données précédentes, lors de l'étude des corrélations simples.

\section{Classification et réduction des données}

L'exploitation des données disponibles (fig. 2) peut s'effectuer suivant les 3 modalités (représentées fig. 3 ).

Dans la mesure oủ la discrimination doit s'effectuer à partir des paramètres physiques et pétrographiques, seules les modalités 1 et 3 sont utilisables, La première tient compte de la totalité de la dispersion de chaque variable alors que l'analyse des moyennes des variables au sein d'une population, est particulièrement intéressante lors de l'étude des corrélations.

Dans une première étape, nous nous intéresserons à la totalité des individus secs et rechercherons une partition stable et les paramètres discriminants.

\subsection{Analyse en composantes sur l'ensemble de l'échantillonnage}

Nombre d'observations $=196 \quad$ Nombre de variables $=7$ Pour clarifier l'exposé, cette analyse sera présentée en détail. Par la suite, les interprétations seront plus rapides mais s'inspireront du même esprit.

Fig. 2 Valeur moyenne des variables étudiées

\begin{tabular}{|c|c|c|c|c|c|c|c|c|c|c|c|}
\hline NOM & $N^{0}$ & $\begin{array}{l}\text { VSE } \\
\mathrm{m} / \mathrm{s}\end{array}$ & $\begin{array}{l}\text { VSA } \\
\mathrm{m} / \mathrm{s}\end{array}$ & $\begin{array}{c}\text { PVS } \\
\mathrm{kN} / \mathrm{m}^{3}\end{array}$ & $\begin{array}{l}W \\
\%\end{array}$ & $\begin{array}{l}\text { RSE } \\
\mathrm{MPa}\end{array}$ & $\begin{array}{l}\text { RSA } \\
\mathrm{MPa}\end{array}$ & $\begin{array}{l}\mathrm{ESA} \\
\mathrm{MPa}\end{array}$ & $\begin{array}{l}\text { ESE } \\
\text { MPa }\end{array}$ & & $\begin{array}{l}\text { RSE } \\
\text { RSA }\end{array}$ \\
\hline RIBECOURT & 1 & 2822 & 2603 & 17.1 & 15,90 & 13,2 & 7 & 2280 & 4690 & 2 & 1.83 \\
\hline HORDAIN 1 & 2 & 1466 & 1168 & 16,4 & 18,60 & 5,2 & 2,4 & 480 & 1000 & 4 & 2,16 \\
\hline HORDAIN 2 & 5 & 2000 & 1527 & 16,8 & 17,70 & 9,6 & 4,5 & 1400 & 1890 & 2 & 2,13 \\
\hline MILLHOMMES & 4 & 2056 & 1705 & 16,5 & 14,51 & 9,8 & 4,9 & 1240 & 2680 & 2 & 2,33 \\
\hline MARLY & 5 & 2585 & 2054 & 17,5 & 12,27 & 14 & 4,7 & 930 & 2830 & 3 & 2,98 \\
\hline VALENCIENNES & 6 & 2089 & 1995 & 16,7 & 16,97 & 9,5 & 4,4 & 1060 & 2050 & 2 & 2,16 \\
\hline ESTREUX & 7 & 2240 & 1371 & 16,8 & 17,31 & 8,2 & 4,6 & 1070 & 170 & 2 & 1,78 \\
\hline St SAULVE & 8 & 2537 & 2332 & 16,4 & 21,91 & 8,9 & 4 & 1250 & 2000 & 3 & 2,22 \\
\hline LEZENNES A & 9 & 2519 & 2362 & 16,2 & 21,69 & 6,9 & 4,3 & 2410 & 2500 & 3 & 1,60 \\
\hline LEZENNES B & 10 & 2629 & 2400 & 16,6 & 20,23 & 9,0 & 4,2 & 2160 & 2990 & 3 & 2,14 \\
\hline VALENC. HIOLLE & 11 & 1753 & 1377 & 17,1 & 17,66 & 5,2 & 2,9 & 690 & 780 & 4 & 1,79 \\
\hline St SAULVE (Pouille) & 12 & 2657 & 2550 & 16,9 & 21,24 & 9,3 & 5,3 & 2360 & 3030 & 3 & 1,75 \\
\hline PETITE FORET & 13 & 2384 & 2240 & 16.5 & 21,56 & 12,7 & 3,8 & 2000 & 4290 & 3 & 3,34 \\
\hline LOOS & 14 & 2542 & 2384 & 15,8 & 21,88 & 6,4 & 4 & 2340 & 2230 & 3 & 1,60 \\
\hline AVESNES-LE-SEC & 15 & 2383 & 2315 & 17,4 & 14,78 & 14,8 & 5,4 & 1670 & 4990 & 2 & 2,74 \\
\hline WATTIGNIES & 16 & 2677 & 2255 & 17,4 & 18,19 & 15,5 & 4,9 & 2270 & 5230 & 3 & 3,16 \\
\hline CAMBRAI GARE & 17 & 2619 & 2489 & 16,4 & 22,15 & 12,3 & 6,4 & 3140 & 4500 & 3 & 2,02 \\
\hline CAMBRAI MARCHE & 18 & 2568 & 2395 & 16,4 & 21,62 & 15 & 4,9 & 2590 & 5260 & 3 & 3,06 \\
\hline LILLE-DOM BOSCO & 19 & 2416 & 2311 & 16,9 & 21,49 & 5,3 & 3,4 & 2280 & 1885 & 3 & 1,56 \\
\hline F. TUMESNIL & 20 & 2397 & 2346 & 15,6 & 22,07 & 5,5 & 4,2 & 1940 & 2000 & 3 & 1,31 \\
\hline F. TUMESNIL Stade & 21 & 2516 & 2433 & 16,2 & 21,85 & 5,7 & 3,9 & 1640 & 2140 & 3 & 1,46 \\
\hline
\end{tabular}

Fig. 3 Les trois modalités de calcul

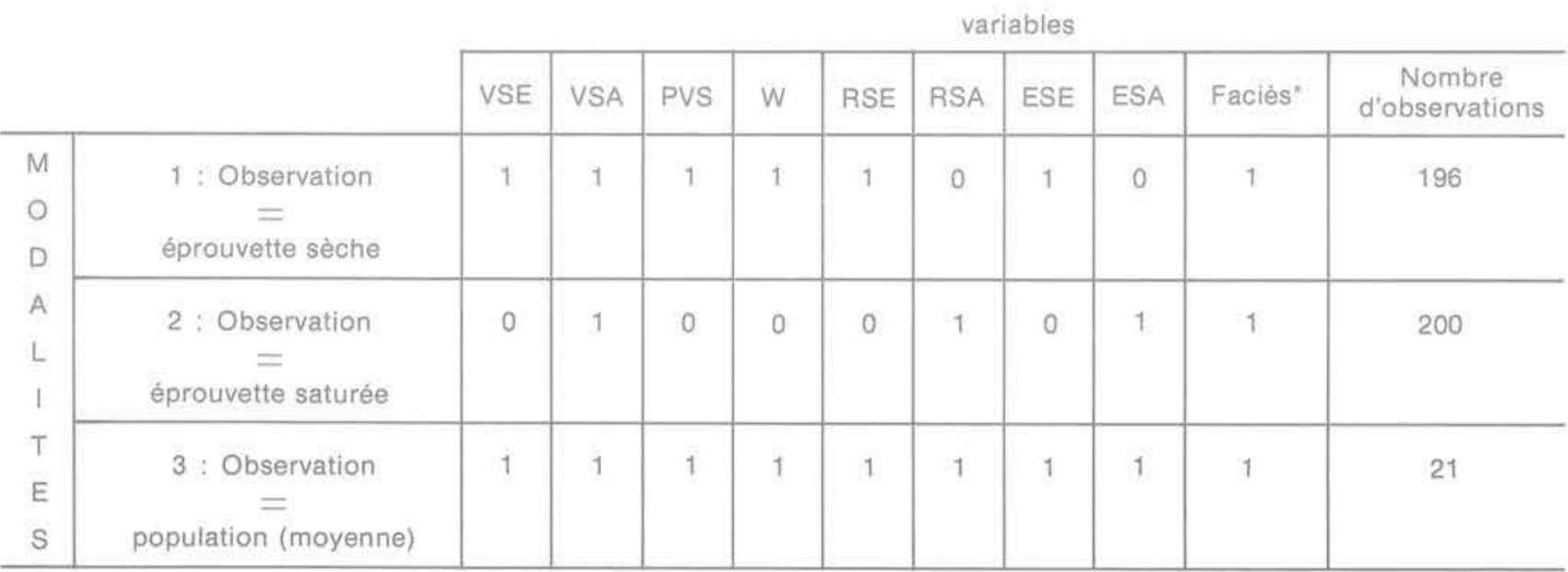

- Variable qualitative à 3 modalités, notées PET2, PET3 et PET4 (pétrographie) 
Les pourcentages d'inertie ou contribution des premiers axes principaux* sont élevés (fig. 4) aussi, nous limiterons l'essentiel de l'interprétation aux nuages projetés sur les premiers plans principaux.

Chaque composante principale est en général le reflet d'une combinaison de plusieurs variables initiales.

L'axe 1 est fortement influencé par les variables VSE et VSA $(-0,83$ et $-0,90)$ et oppose les craies blanches à l'ensemble des craies glauconieuses.

L'axe 2 est corrélé négativement avec les variables mécaniques RSE et ESE elles-mêmes bien liées ensembles et les variables physiques relatives au degré de compaction ou de lithification du matériau: teneur en eau et poids volumique. L'axe 3 (A3) dont le pourcentage d'inertie est faible (12,5\%) est le résultat d'une combinaison de l'ensemble des variables parmi lesquelles PET2 $(-0,56)$ joue le rôle le plus notable.

La matrice des corrélations initiales (fig. 6) fait apparaître un certain nombre de liaisons intéressantes notamment entre VSE et VSA ou RSE et ESE. Toutefois, les coefficients de corrẻlation sont généralement faibles, ce qui traduit l'hètérogénéité de l'échantillonnage.

Les projections des observations dans les plans principaux A1-A2 et A2-A3 vont faciliter l'interprétation.

Dans le premier plan principal (A1-A2), la projection du nuage est morcelée et disjointe (fig. 7), cette partition prend tout son sens lorsqu'il est fait référence à la nature pétrographique des faciès considèrés.

Nous distinguerons 3 groupes principaux, tous orientés parallèlement á RSE, du moins à la projection de son vecteur unitaire dans le premier plan principal.

- la famille des craies glauconieuses corrélée positivement avec A1 et fortement discriminée par les variables constitutives de $\mathrm{A} 2$, notamment les variables mécaniques. Plus la teneur en glauconie croit, plus les caractéristiques mécaniques chutent.

- Ia famille des craies blanches, à l'exception du site de Marly est un nuage compact comportant également 2 póles à caractéristiques très différentes, Loos et Wattignies, ce qui peut être mis en relation avec des propriẻtés physiques très dissemblables (PVS notamment).

- une partition mixte, composée d'un faciès de craie glaunieuse (Ribecourt) et de la population des craies blanches manquant à l'ensemble précédent (Marly).

- Chaque axe principal ou axe d'inertie est associé à un vecteur propre de l'espace des variables.

Fig. 4 Contribution des quatre premières composantes à l'explication de la variation

\begin{tabular}{c|c|c}
\hline Axe & $\%$ d'inertie & $\%$ d'inertie cumule \\
\hline 1 & 40.01 & 40.01 \\
2 & 31.05 & 71.06 \\
3 & 12.52 & 83.58 \\
4 & 7.03 & 91.51 \\
\hline
\end{tabular}

Cette dernière partition est en fait discriminée dans le troisième plan principal (fig. 8) Ribecourt est corrélé négativement avec $\mathrm{A} 3$ alors que les craies blanches de Marly le sont positivement et par lả-même s'agglomèrent au nuage de la famille des craies blanches lui-même fortement discriminé par l'axe 2

La famille des craies blanches bien groupée dans le premier plan principal apparaît très étalée dans cette deuxième projection et, particulièrement, discriminèe par les variables mécaniques ESE, RSE mais aussi par PVS.

\section{Conclusion de l'analyse en composantes principales}

L'analyse conduit à la discrimination de 2 grandes familles. elles-mêmes relativement morcelées suivant la projection considérée.

La famille des craies glauconieuses qui comporte 74 individus provenant de 8 sites. Le nuage constitué par ces observations est bien discriminé par les variables vitesse du son et comporte 2 grands pôles :

- le pôle "Ribecourt-Avesnes" à vitesse du son élevée et fortes caractéristiques mécaniques, oú la glauconie peu abondante est disséminée dans la matrice crayeuse sous la forme de petits nodules à peine perceptibles à l'œil nu (craie piquetée).

- le pôle "Ernest Hiolle" oú la glauconie est individualisée en feuillets visibles à l'œil nu, caractérisé par une vitesse du son faible et de mauvaises caractéristiques mécaniques.

La zone médiane, composée de 49 individus, est tout-à-fait intermédiaire, tant du point de vue texture que du point de vue mécanique.

La famille des craies blanches constitue un nuage plus compact, évoluant plutôt parallèlement à l'axe 2 et corrélé négativement avec $\mathrm{A} 1$. II est cependant probable que des partitions internes peuvent se dessiner, sous l'influence des variables constitutives de l'axe 2 : teneur en eau et poids spécifique. Cette discrimination apparaît d'ores et dèjà dans le $1^{\text {er }}$ plan principal oủ se distingue notamment le site de "Marly".

H apparaît clairement que les variables physiques les plus discriminantes ne sont pas identiques pour ces deux familles, ce qui explique les coefficients de corrélation relativement faibles (entre paramètres mécaniques et paramètres physiques).

Fig. 5 Caractéristiques générales des craies glauconieuses. Corrélation. Variables : axes d'inertie

\begin{tabular}{l|r|r|r|r}
\hline \multicolumn{1}{c|}{ AXES } & 1 & \multicolumn{1}{c|}{2} & \multicolumn{1}{c}{3} & \multicolumn{1}{c}{4} \\
\hline 1 VSA & -.9006 & -.1908 & -1275 & 1656 \\
2 VSE & -.8341 & -.3365 & -1667 & 3116 \\
3 W & -.5627 & 6457 & .0173 & -3265 \\
4 PSP & 2737 & -.7113 & .3787 & 4347 \\
5 RCS & -.2466 & -.8128 & .3272 & -3177 \\
6 ESE & -.5216 & -.6656 & .2832 & -3815 \\
7 PET 2 & .5409 & -.5891 & -.5644 & -1450 \\
8 PET 3 & -.8285 & .4002 & .2382 & .1286 \\
9 PET 4 & 6249 & .3331 & .6134 & .0225 \\
\hline
\end{tabular}

Fig. 6 Analyse en composantes principales. Ensemble des craies du Nord ( $N=196)$. Matrice des corrélations initiales

\begin{tabular}{|c|c|c|c|c|c|c|c|c|c|}
\hline & VSA & VSE & W & PVS & RCS & ESE & PET 2 & PET 3 & PET 4 \\
\hline $\begin{array}{l}\text { VSA } \\
\text { VSE } \\
\text { W } \\
\text { PVS } \\
\text { RCS } \\
\text { ESE } \\
\text { PET } 2 \\
\text { PET } 3 \\
\text { PET } 4\end{array}$ & 1. & 1.90006 & $\begin{array}{r}38649 \\
15559 \\
1 . \quad\end{array}$ & $\begin{array}{r}-.07230 \\
.05331 \\
-.64862 \\
1 .\end{array}$ & $\begin{array}{r}23820 \\
.31842 \\
-\quad 30189 \\
.48232 \\
1 .\end{array}$ & $\begin{array}{r}52333 \\
.50224 \\
-.01659 \\
.26312 \\
.82283 \\
1 . \quad\end{array}$ & $\begin{array}{r}-.29165 \\
-.19326 \\
-.59549 \\
.31607 \\
.18804 \\
.01204 \\
1 .\end{array}$ & $\begin{array}{r}58259 \\
.51417 \\
.61725 \\
-.37938 \\
-.04399 \\
.16111 \\
-87307 \\
1 .\end{array}$ & $\begin{array}{r}-61125 \\
-.66373 \\
-.09242 \\
-15547 \\
-.27556 \\
-34865 \\
-17484 \\
1 .\end{array}$ \\
\hline
\end{tabular}




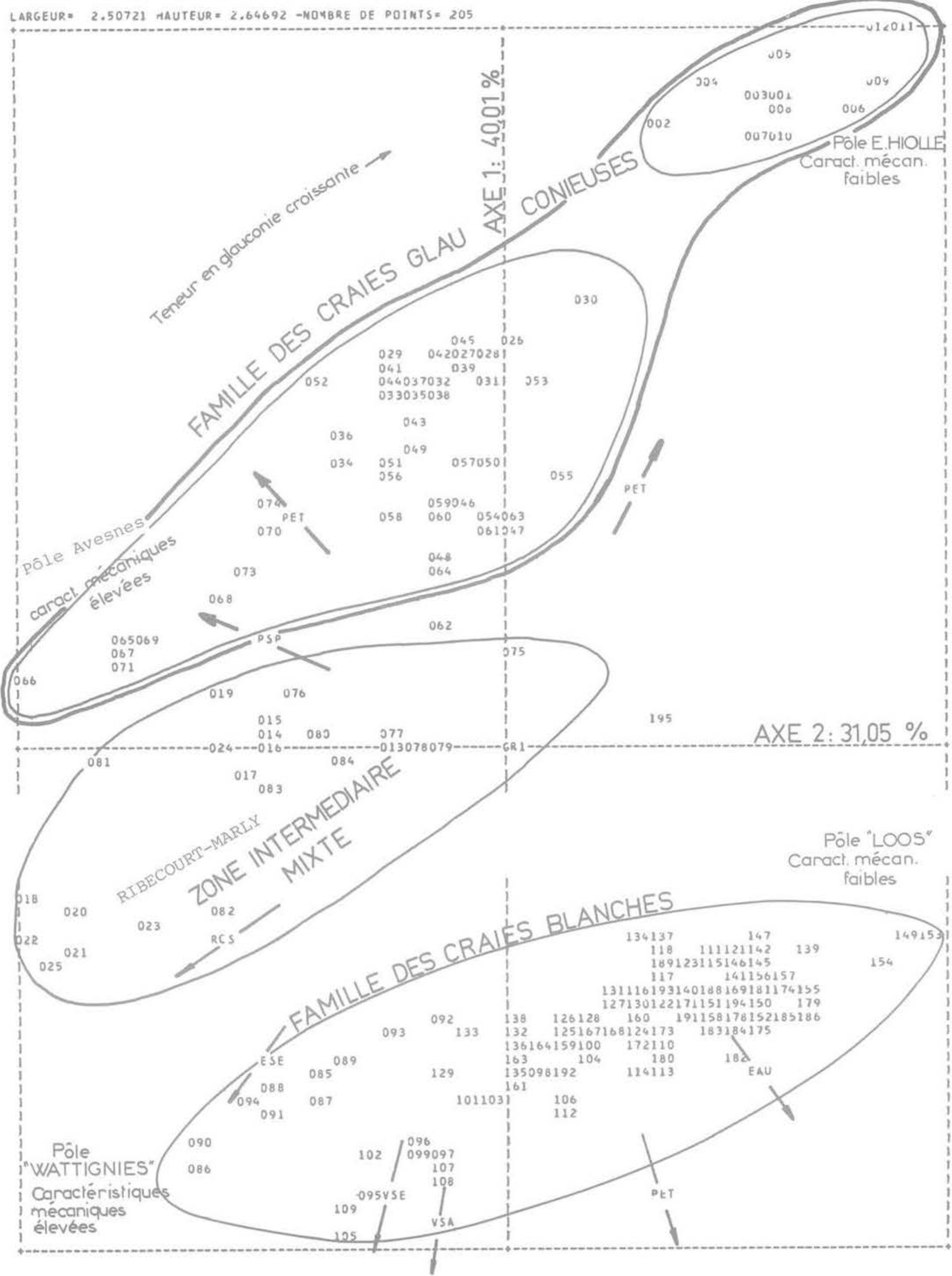

Fig. 7 Projection des observations dans le premier plan principal. Ensemble de l'échantillonnage 
AXE HOKILINTALI 3)--AXE VERTICALI 2) - TITRE:ANALYSE EN COMPJSANTES PRINCIPALES ENSEMBLE DES CRAIES (NQRU). MEI9O. LARGEUR $=\$ .63171$ TAUTEUR $=2.50721$-NOYGRE UE POINTS $=205$

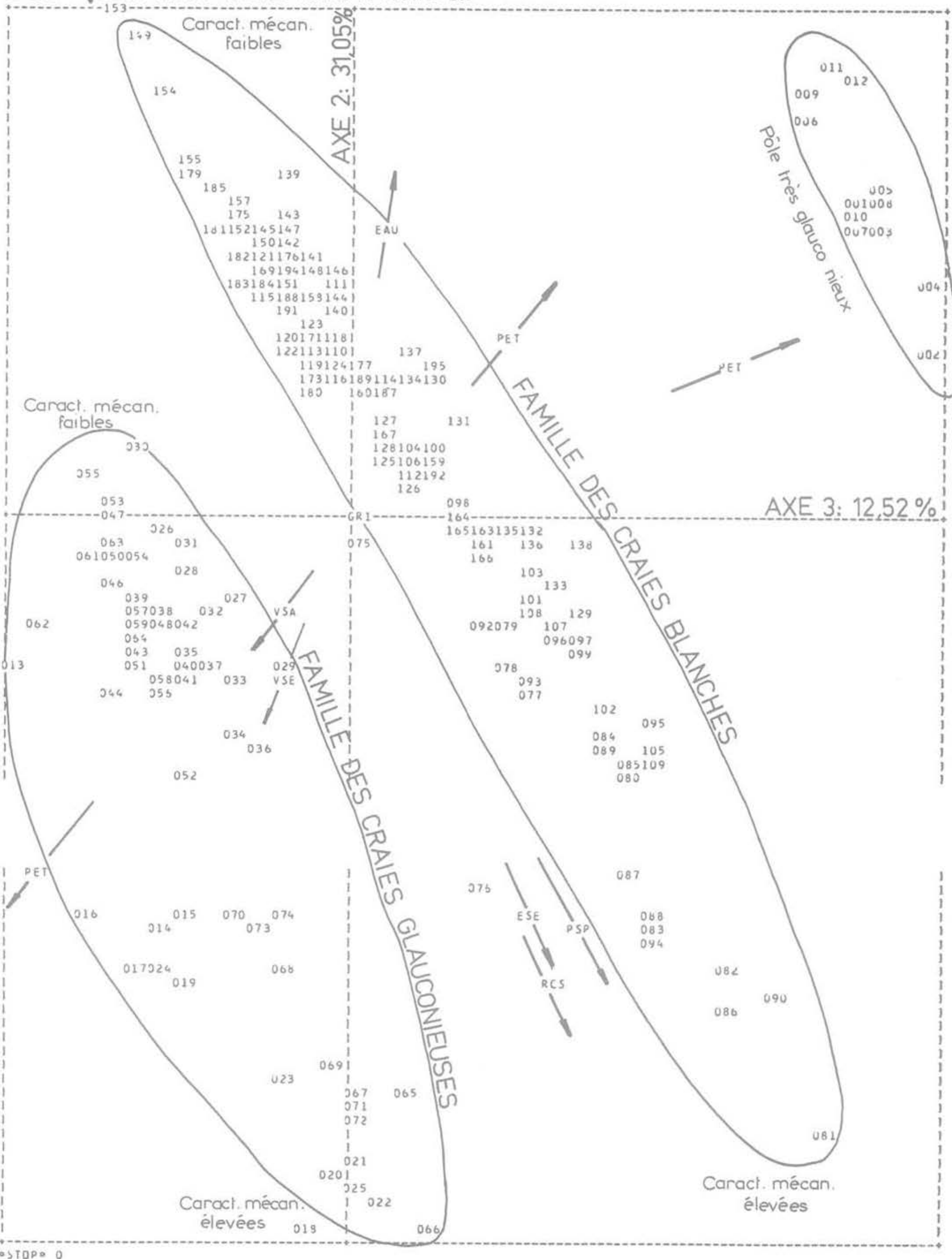

Fig. 8 Projection des observations dans le troisième plan principal. Ensemble de l'échantillonnage 


\section{Stabilité de cette partition}

Cette discrimination est particulièrement stable dans la mesure où elle est conforme aux résultats trouvés par la méthode des nuées dynamiques, (les noyaux les plus stables ètant générés par la variable vitesse du son) et par l'analyse factorielle discriminante qui aboutit à la même classification, les variables les plus discriminantes étant, par ordre décroissant: VSA, W, PVS, VSE, RSE, ESE.

Les axes discriminants sont le reflet d'une combinaison oủ les variables sont affectées de coefficients permettant de juger du "poids" de chaque variable.

L'axe factoriel F1 qui a un pouvoir discriminant de $86 \%$ est essentiellement celui de la vitesse du son alors que l'axe F2 $(14 \%)$ représente le poids volumique sec.

\section{Analyse des moyennes}

La discrimination à laquelle nous sommes arrivés repose sur les échantillons testés à l'état sec. L'influence des paramètres mécaniques RSA et ESA va pouvoir être étudiée en analysant en composantes principales les moyennes des variables relatives à chaque population (modalité 3 ). Les pourcentages d'inertie des 2 premiers axes principaux font respectivement $49,46 \%$ et $30,99 \%$. Aussi, la seule projection dans le $1^{\text {or }}$ plan principal $(80,45 \%)$ suffit à l'analyse.

Dans l'espace des variables (fig. 9) toutes les variables sont corrélées positivement avec A1, surtout les variables mécaniques et les variables vitesse du son, elles-mēmes bien corrélées entre elles.

Cette première composante est logiquement liée à la soliditè des échantillons.

L'axe 2 oppose le poids spécifique et l'indice de porosité. c'est un indicateur de compaction, autrement dit de degré de diagènèse. On notera que RSE est également corrélé positivement avec cet axe $(0,58)$ ce qui est tout à fait comprèhensible.

En conclusion, il apparaît que les paramètres discriminants sont différents suivant les familles ètudiées. Aussi la deuxième étape reposera-t-elle sur l'analyse parallèle de ces 2 familles en utilisant des méthodes similaires.

\subsection{Analyse en composantes principales des craies glauconieuses}

Le nombre d'observations est 74 , le nombre de variables de 7. Le pourcentage d'inertie de A1 est remarquablement èlevé $(61,78 \%)$ par rapport aux pourcentages de $A 2$ et $A 3$ qui sont voisins de 15 et $13 \%$ (fig. 10).

L'axe 1 est fortement corrélé avec VSE (plus de 0,92) et les variables mécaniques RSE et ESE $(0,85$ et 0,91$)$. c'est donc l'axe de la résistance. Au contraire, les axes 2 et 3 ne sont corrélés respectivement qu'avec une seule variable, la teneur en eau et le poids spécifique. Une projection du nuage des observations dans le deuxième plan principal fait apparaître les 3 familles qui jusqu'à présent ne constituaient que des pôles. Cette discrimination s'effectue essentiellement le long de l'axe 1. II est donc probable que la seule mesure de la vitesse du son suffit pour caractériser ce type de craie. Des analyses plus fines portant sur ces partitions ont été effectuées mais elles n'ont pas mis en évidence d'autres facteurs intèressants.

\subsection{Analyse de la famille des craies blanches}

Nombre d'individus $=121$ Nombre de variables $=6^{*}$ L'analyse en composantes principales montre que A1 caractèrise le bon comportement mécanique et un degré de lithification élevé (PVS est corrélé à 0,84 avec A1), alors que A2 et A3 concernent la texture (VL, W). Par construction, les composantes principales sont indépendantes, aussi VSA mais également VSE ne sont pas du tout corrélées avec les caractéristiques mécaniques. D'ailleurs cette présomption est corroborée par les résultats de l'analyse factorielle dis-

- La variable pétrographique est supprimee car elle n'a qu'une modalité.

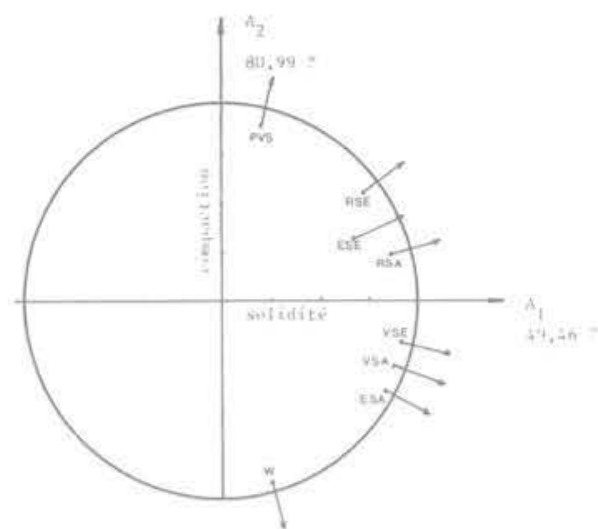

Fig. 9 Analyse en composantes principales ; cercle des corrélations dans le premier plan principal. Totalité de l'échantillon

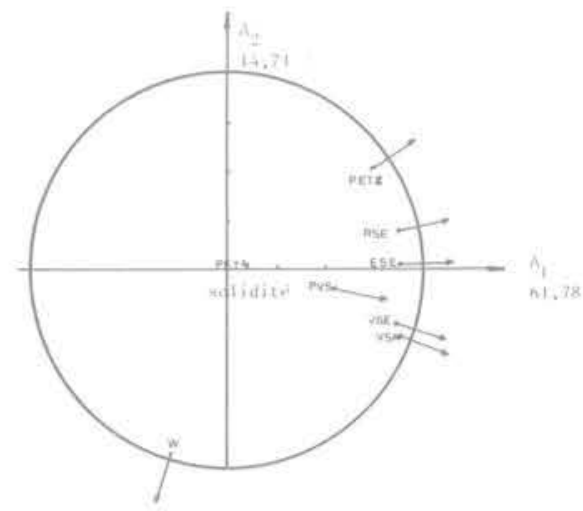

Fig. 10 Analyse en composantes principales; cercle des corrélations dans le premier plan principal. Craies glauconieuses

criminante qui met en évidence le pouvoir discriminant de chaque paramètre : la seule variable W, classe $97 \%$ des observations (en fonction de leurs appartenances aux 3 groupes décelés précédemment, à savoir : Marly - Wattignies - le reste de craies blanches). Aussi, les corrélations simples entre résistance à la compression à l'état saturé et indice de porosité seront particulièrement étudiées. 
CRAIES GLAUCONIEUSES

(Grises, vertes...)

\begin{tabular}{l|c|c|c|c|c} 
Glauconie visible à \\
I'œil nu, disposée \\
en feuillets bien \\
individualisès
\end{tabular}

Fig. 11 Caractéristiques générales des craies glauconieuses

\section{Etude des corrélations entre paramètres physi- ques discriminés et variables mécaniques}

\subsection{Etude des craies glauconieuses}

La classification retenue comporte 3 groupes dont les caractéristiques générales sont présentées dans le tableau de la figure 11. VSE et VSA sont les parametres physiques qui varient dans l'intervalle le plus grand:

de 1705 a $2808 \mathrm{~m} / \mathrm{s}$ pour VSE (moyenne)

de 1300 à $2606 \mathrm{~m} / \mathrm{s}$ pour VSA (moyenne)

avec un coefficient de variation très faible au sein de chaque famille

$$
\mathrm{cv}=\frac{\sigma}{\mathrm{m}}=0,07
$$

L'étude des corrèlations simples peut s'effectuer à partir des observations (74) ou des moyennes des populations constituant la famille. Pour s'affranchir de la dispersion due à l'essai nous avons travaillé à partir des moyennes $(n=80 u$ 9). Les coefficients de corrélation impliquant les paramètres mécaniques et la vitesse du son figurent dans le tableau suivant :

\begin{tabular}{l|c|c|c|rl}
\hline & RSE & RSE & ESE & ESA \\
VSE & 0,9866 & 0,8281 & 0,8060 & 0,9369 & $\rho$ \\
& $97,34 \%$ & $68,57 \%$ & $64,96 \%$ & $87,78 \%$ & $\rho^{2}$ \\
\hline VSA & 0,9330 & 0,8876 & 0,8705 & 0,8647 & $\rho$ \\
& $87,05 \%$ & $78,79 \%$ & $75,76 \%$ & $74,76 \%$ & $\rho^{2}$ \\
\hline
\end{tabular}

$\rho^{2}=$ coefficient de corrélation

$\rho^{2}=$ pourcentage de la variance expliqué

Fig. 12 Tableau des coefficients de corrélation et pourcentage expliqué
Les meilleures liaisons relatives aux paramètres mécaniques saturés sont représentées sur la figure 13. II apparaît clairement que la seule connaissance de VSE permet de prévoir le comportement mécanique des craies glauconieuses, notamment à l'état saturé, avec une finesse suffisante. Ces courbes permettent l'évaluation des parametres mécaniques moyens et de la dispersion relative à chaque famille. Sur les graphes, il a été représenté une estimation de cette dispersion (1 écart type de part et d'autre de la moyenne). A la vitesse VSE $=2200(\rho=200 \mathrm{~m} / \mathrm{s})$, par exemple, correspond une valeur moyenne de 4,8 MPa avec un écart type de 1,2 $\mathrm{MPa}$.

La dispersion proposée correspond à un coefficient de variation $\mathrm{cv}=\frac{\sigma}{\mathrm{m}} \neq 0,22$. Elle a été calculée à partir de populations comprenant plus de 12 individus. Les expériences parallèles que nous avons effectuées sur des craies, comportant une trentaine d'individus, ainsi que les valeurs disponibles dans la littérature nous incitent à penser qu'une valeur de cv comprise entre 0,20 et 0,30 est réaliste, suivant la sensibilité du matériau á l'humidité.

\subsection{Etude des craies blanches}

Les analyses précédemment effectuées sur les craies blanches font apparaître des partitions stables oủ le poids spécifique et la porosité sont les variables les plus discriminantes. L'étude des liaisons entre ces paramètres et les grandeurs mécaniques mesurées tant à l'état sec qu'à l'état saturé n'a pas mis en évidence de corrélation statistiquement utilisable. Le coefficient de corrélation le plus élevé $(0.77)$ caractérise la liaison PVS - RSE, ce qui explique $59 \%$ de la variance. Mais, le degré de saturation du matériau "in situ" est proche de $100 \%$ aussi le paramètre essentiel est-il RSA. Cette grandeur est d'une remarquable stabilité et varie entre 3,4 et $6,4 \mathrm{MPa}$, la grande majorité des sites (10 sur 13) se situant entre 3.8 et $4,9 \mathrm{MPa}$ avec des écarts types tels que les différences ne sont pas significatives.

D'une manière générale, les craies à fort poids spécifique $(P V S \simeq 1,74)$ ont des caractéristiques sèches élevées. Les 


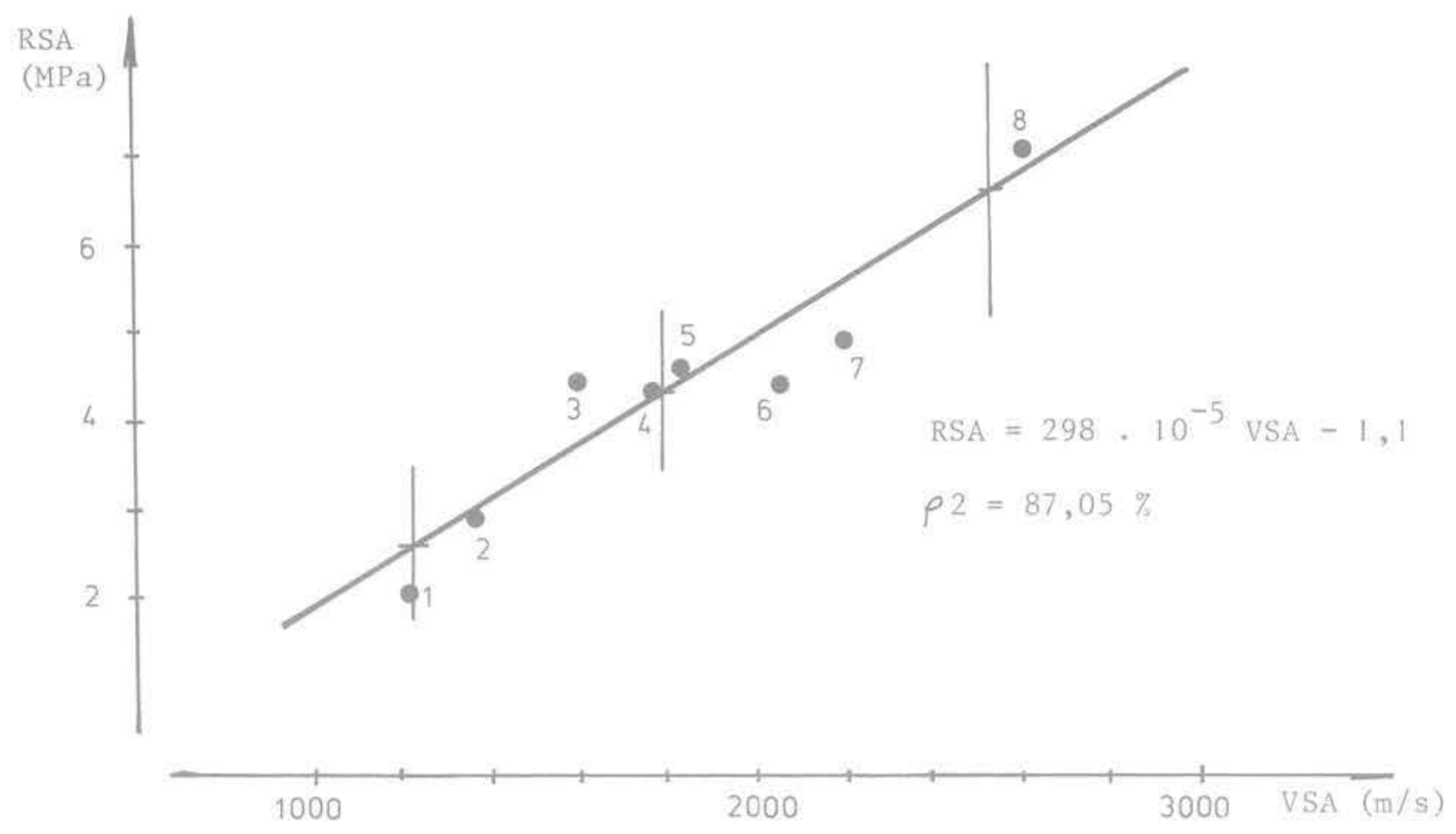

Liaison RSA - VSE

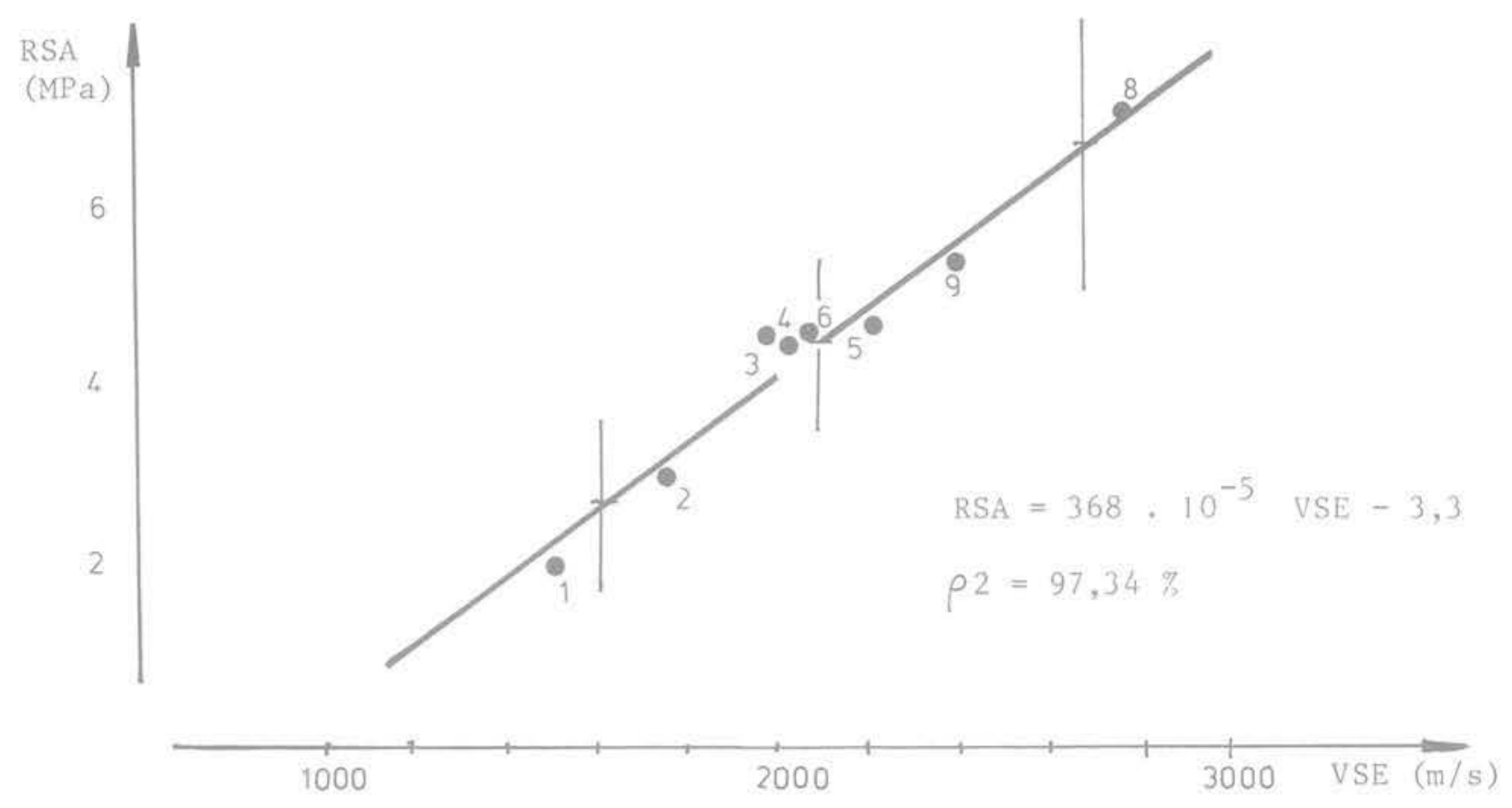

Fig. 13 Liaison résistance-vitesse 
craies blanches du Turonien supérieur (Marly) se rattachent à cette famille. Cependant, l'inverse n'est pas vrai et certains faciès du Cambrésis ayant des poids volumiques de 1,64 résistent en moyenne à 12 ou $15 \mathrm{MPa}$ avec de forts modules.

Les craies blanches sénoniennes, notamment dans les régions de Lille et de Valenciennes ont des caractéristiques sèches moins élevées (en général RSE $<10 \mathrm{MPa}$ ) mais des caractéristiquessaturées très constantes. Pour ce type de faciès, la valeur moyenne de RSA se situe autour de 4,2 MPa avec un écart type de $1 \mathrm{MPa}$.

\section{Conclusions}

Dans le cadre d'une étude de stabilité sommaire, il est possible d'estimer les caractéristiques mécaniques du matériau avec une précision jugée suffisante. En ce qui concerne les craies glauconieuses, les valeurs moyennes de la vitesse des ondes longitudinales, dans le matériau sec, bien corrélées avec la résistance à la compression à l'état sec, sont directement opérationnelles. Au sein des craies blanches du Nord, les paramètres physiques considèrés n'autorisent pas de conclusions quantitatives mais ces faciès ont des qualités mécaniques relativement constantes à l'état saturé, généralement voisines de $5 \mathrm{MPa}$.

En revanche, lors d'une étude plus fine ou lorsque le coefficient de sécurité semble proche de 1, il est nécessaire de prendre en compte la dispersion du matériau et l'expérimentateur ne peut pas s'affranchir d'essais en laboratoire, notamment au niveau des zones les plus sollicitées, comme les piliers.
Remerciements : Nous remercions les ingénieurs du Cerchar pour leur collaboration et tout particulièrement Monsieur Dejean pour ses nombreux conseils concernant les techniques d'analyse des données.

\section{Références Bibliographiques}

[1] COMES G. - AKERMANN G. (1976) - La craie et la réfrigération des centrales électronucléaires situées sur le littoral. Comité Français de Géologie de I'Ingénieur. Rouen.

[2] BONVALLET J. (1978) - Critères de stabilité des exploitations souterraines à faible profondeur. Application au cas des carrières souterraines du Nord. Thése NancyINPL.

[3] DARCY J. (1976) - Exemple d'un tunnel routier dans la craie. Comité Français Géologie de I'Ingénieur. Rouen.

[4] DESSENNE J.L. - DUFFAUTP. (1970) - Les propriétés rhéologiques de la craie et leur influence sur le percement des galeries. La Houille Blanche. № 5 p. 477 - 487.

[5] DESSENNE J.L. (1971) - Etude rhéologique et géotechnique de la craie. Thèse Grenoble.

[6] TALLON J.P. - Influence de la texture sur certaines propriêtés géotechniques des craies franches. Thèse de Docteur Ingénieur. Paris VI 1976. 